um die Regressionsgerade III (normaler Gentypus) verteilen. Die aus den gemessenen Werten der atypischen $\mathrm{SChE}$ errechnete Regressionsgerade $\mathrm{V}$ ist signifikant gegen die Regressionsgerade III verschieden (Abb. 11).
Bei der atypischen SChE ist die parallel mit dem AclsolestTestpapier gemessene Aktivität deutlich tiefer gegenüber dem Normaltypus.

\title{
Literatur
}

1. Kalow, W. und H. A. Lindsay, Canad. J. Biochem. Physiol. 33, 568 (1955). - 2. Kalow, W. und K. Genest, Canad. J. Biochem. Physiol. 35, 339 (1957). - 3. Saller, S. und H. BraunsteINER, Klin. Wschr. 37,986 (1959). - 4. RICHTERICH, R., Schweiz. med. Wschr. 92, 263 (1962). - 5. Churchill-Davidsov, H. C. und W. J. Grifriths, Brit. Med. J. 14, 994 (1961). - 6. Ammon, R., Pflügers Arch. ges. Physiol. Menschen Tiere 233, 486 (1933). - 7. LANG, W. und G. Intsesuloglu, Klin. Wschr. 40, 312 (1962). - 8. Glick, D., J. biol. Chemistry 137, 357 (1941). - 9. JABsA, Z.,

M. Schönfelder und H. Breuer, Klin. Wschr. 39,966 (1961). - 10. Micher, H. O., J. Laborat. Clin. Med., S. Louis 34, 1564 (1949). 11. Pilz, W. und H. Höruerrs, Hoppe-Seyler's $Z$. physiol. Chem. 339157 (1964). - 12. Doenicke, A. und St. Schimidinger, Med. Klinik, 60, 2012 (1965). - 13. Doenicke, A., Th. Gürthiner, J. Remes, W. Spiess, K. Steinbereitihner und E. Vonkilci, Wien, Proc. II 191 (1962); 106. Colloquium über Narkose und Anästhesie Hamburg 23. 1. 1963. - 14. Steinbereitriner, K., Wien. klin. Wschr. 76, 785 (1964).

Priv.-Doz. Dr. A. Doenicke

Chirurg. Univ.-Poliklinik

8 München 15, Pettenkoferstr. 8 a

\section{Beeinflussung der Erythrozyten-Sedimentationsrate und der enzymatischen Neuraminsäure-Abspaltung durch Zusatz eines kleinmolekularen Glykoproteids}

Herrn Professor H. PlügGe zum 60. Geburtstag gewidmet

Von H. WeICKer und D. KuHN ${ }^{1}$ )

Aus der Abteilung für Stoffuechselforschung (Leiter: Prof. H. Weicker) der Medizinischen Universitäts-Poliklinik Heidelberg (Direktor: Prof. H. Plïgge)

(Eingegangen am 22. Januar 1966)

Die Beeinflussung der Erythrozyten-Sedimentationsrate durch ein neuraminsäurereiches, kleinmolekulares Glykoproteid ging der Konzentration des zugesetzten Glykoproteids direkt parallel. Seine chemischen und physikalischen Eigenschaften entsprechen denjenigen eines kleinmolekularen $\alpha_{1}$-Serum-Glykoproteids. Nach schonender enzymatischer Abspaltung der Erythrozyten-Neuraminsäure mit Neuraminidase wurde dieser Effekt noch verstärkt; wurde die Glykoproteid-Neuraminsäure vor Zusatz zu dem Plasma-Blut enzymatisch abgespalten und die nicht entfernbare Neuraminidase durch Hitze inaktiviert, dann war der Anstieg der Sedimentationsrate etwas geringer. Hingegen war bei nicht hitzeinaktiviertem Glykoproteid nach Neuraminidase-Behandlung die Senkungszunahme noch ausgeprägter. Durch diesen Glykoproteid-Komplex wurde die Virus-Haemagglutination total gehemmt. Wurde anstelle eines Glykoproteids ein Glykolipid (Gangliosid) zugesetzt, dann war nach Abspaltung der Neuraminsäure kein senkungsbeschleunigender Effekt mehr festzustellen. Wurde das Plasmavolumen bei konstanter Protein-Konzentration vergrößert, trat eine Erhöhung der Sedimentationsrate ein. Nach Einengen des Plasmas war der senkungsbeschleunigende Effekt bei glykoproteidreichem Plasma größer als bei Normalplasma oder Plasma mit $\boldsymbol{\gamma}$-GlobulinVermehrung. Die Zunahme des Erythrozytenvolumens, wie es bei Eindickung oder Polyglobulie vorliegt, verminderte die Sedimentationsrate durch relative Verminderung der negativen Plasmaladung pro Erythrozyt. Hydrämien mit Reduktion der Proteinkonzentration im Plasma lösten ebenfalls eine Verminderung der Blutsenkungsgeschwindigkeit aus. Enzymatischer Release der Erythrozyten-Neuraminsäure zeigte bei schonender Inkubation mit Neuraminidase eine Steigerung der Erythrozyten-Agglomerisation. Bei komplettem Neuraminsäure-Release hingegen fehlte die Erythrozyten-Agglomerisation. Der enzymatische Neuraminsäure-Release lag zwischen $80-90 \mu \mathrm{g}$ pro $\mathrm{ml}$ gepackte Erythrozyten.

The change in the sedimentation rate of erythrocytes is directly proportional to the concentration of a low molecular weight glycoprote in, which is rich in neuramic acid. Its chemical and physical properties correspond to those of a small molecular weight $\alpha_{1}$-serum glycoprotein. After the mild enzymic remofal of the erythrocyte neuramic acid with neuramidase, this effect was intensified. It the neuramic acid of the glycoprotein was removed enzymically and the non-removable neuramidase inactivated by heating prior to the addition to the serum, then the increase in the sedimentation rate was somewhat lower. When the heat inactivation was omitted, however, the increase in sedimentation was more marked. Virus haemagglutination was totally inhibited by this glycoprotein complex. If the glycoprotein were replaced by a glycolipid (ganglioside), then, after, removal of the neuramic acid, there was no longer any acceleration of sedimentation. An increase in plasma volume with constant protein concentration resulted in an increase in the sedimentation rate. After reducing the plasma volume, the sedimentation accelerating effect was larger in plasma rich in glycoprotein than in normal plasma or plasma with increased $\gamma$-globulin. The increase of the erythrocyte volume, as in inspissation or polyglobulism, decreased the rate of sedimentation by relatively decreasing the negative plasma charge per erythrocyte. Hydraemia with reduction of the plasma protein concentration also caused a decrease in the rate of erythrocyte sedimentation. The enzymic release of the erythrocyte neuramic acid when carefully incubated with neuramidase was accompanied by an increase in erythrocyte agglomeration. The complete release of neuramic acid, however, gave no erythrocyte agglomeration. The enzymic release of neuramic acid was 80-90 $\mu \mathrm{g}$. per $\mathrm{ml}$. of packed erythrocytes.

1) Mit Unterstützung der Deutschen Forschungsgemeinschaft. 
Dic Erythrozyten-Senkungsreaktion hat trotz der Anwendung moderner Verfahren zur Differenzierung der Serum-Eiwcißkörper wie Elektrophorese, Ultrazentrifuge und Gel-Filtration ihre Bedeutung als Routinemethode für Klinik und Praxis nicht verloren. Obwohl der Senkungsmechanismus in zahlreichen experimentellen Arbeiten intensiv untersucht wurde, ist er im einzelnen noch nicht vollständig aufgeklärt. In dieser Mitteilung soll durch Modellversuche mit einem kleinmolekularen neuraminsäurereichen Glykoproteid die Glykoproteid-Einwirkung auf den Senkungsablauf dargestellt werden. Da die Neuraminsäure sowohl vom Erythrozyten als auch von dem Glykoproteid enzymatisch abspaltbar war, konnte der Einfluß der proteingebundenen Neuraminsäure des Glykoproteids und der Erythrozyten-Membran isoliert untersucht werden. Außerdem wurde die Bedeutung der ErythrozytenPlasma-Relation und der Hydrämie auf den Senkungsablauf gezeigt. Hierbei ergaben sich neben praktisch wichtigen Gesichtspunkten auch theoretische Befunde, die zur Klärung des Senkungsmechanismus beitragen konnten.

\section{Methodik}

Isolierung des Glykoproteids: siebe Tabelle 1

Versuchsansätze zur Beeinflussung des Senkungsmechanismus

a) Normalsenkung nach der üblichen Technik, wobei die Senkungsröhrchen - mit aqua dest. gereinigt - vollkommen trocken waren und streng vertikal aufgestellt wurden. Ablesen der Blutsenkung bei Zimmertemperatur nach 30, 60, 120 und 180 Min.

b) Waschen der Erythrozyten mit physiologischer Kochsalzlösung bis der Überstand proteinfrei war, danach Ansetzen der Senkungsreaktion mit verschiedenen Erythrozyten-Plasma-Relationen. Weiterhin wurden Plasma-Verdünnungen mit physiologischer Kochsalzlösung vorgenommen. Nach Druckfiltration des Plasmas bis zur doppelten Konzentration des Serum-Eiweißgehaltes wurde die Senkungsreaktion in einem Erythrozyten:Plasma-Verhältnis von $1: 1$ angesetzt.

Folgende Substanzen wurden dem Plasma 30 Min. vor Senkungsbeginn zugesetzt

a) Glykoproteid (Glp)

b) Glykoproteid (GlpND): Inkubation des Glykoproteids mit Neuraminidase ( $\mathrm{Fa}$. Behring-Werke, Marburg) Substrat:Enzym-Verhältnis von 10:1, 12 Stdn. bei $37^{\circ}, \mathrm{pH} 5,7$. Anschließende Dialyse der abgespaltenen Neuraminsäure und Gefriertrocknung des RestGlykoproteids.

c) Glykoproteid (GlpND $100^{\circ}$ ): nach ND-Abbau Erhitzen auf $100^{\circ}$ zur Inaktivierung der Neuraminidase.

d) Neuraminidase (ND): erschöpfend dialysiert und gefriergetrocknet.

e) Neuraminidase $\left(N D 100^{\circ}\right)$ : Erhitzen der dialysierten Neuraminidase, Dialyse und Gefriertrocknung.

f) Gangliosid ( $G G l I)$ : isoliert aus Rinderhim, wurde über KieselgelSäule fraktioniert ${ }^{1}$ ). Diese Gangliosid-Fraktion enthält keine durch Neuraminidase abspaltbare Neuraminsäure.

g) Gangliosid (GGII ND): Inkubation von GGII mit Neuraminidase unter den oben angeführten Versuchsbedingungen.

h) Gangliosid ( $G G l I I)$ : diese Fraktion enthält ein Molekül Neuraminsäure, das enzymatisch abspaltbar ist.

i) Gangliosid (GGl II ND): Präparation wie bei b)

1) Die Präparation wurde uns freundlicherweise von dem MaxPlanck-Institut für medizinische Forschung, Heidelberg (Direktor: Prof. Dr. R. KuHN) überlassen.
Tab. 1

Aufarbeitungsschema des dialysierten Urins

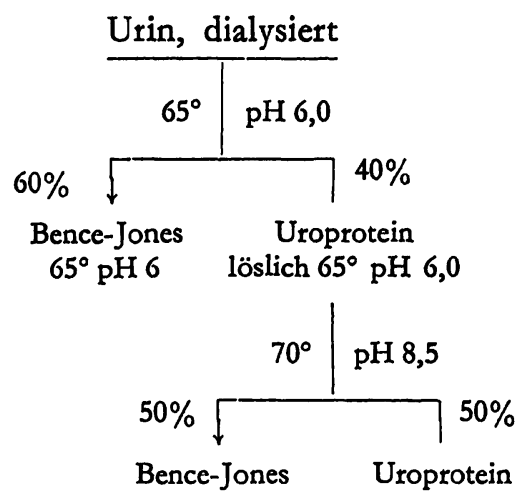

$70^{\circ} \mathrm{pH} 8,5$ löslich $70^{\circ} \mathrm{pH} 8,5$

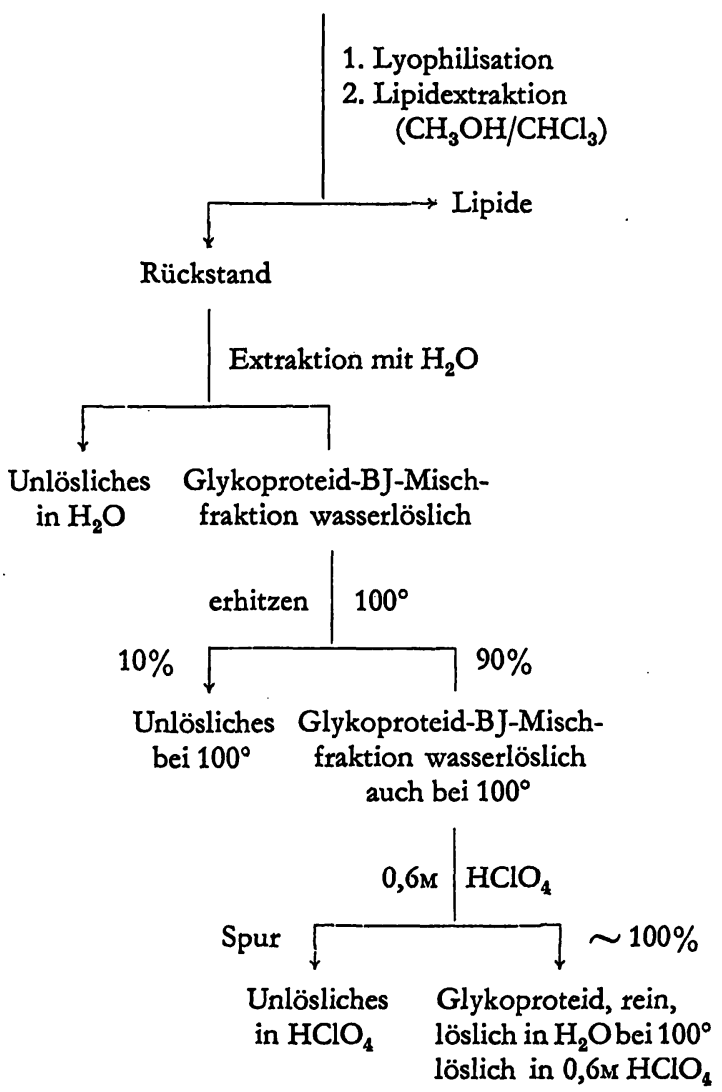

Versuchsansätze zur Ermittlung der Sedimentationsrate

Folgende Senkungsreaktionen wurden angesetzt:

a) Erythrozyten in Plasma

b) Erythrozyten in Plasma +10 bzw. $20 \mathrm{mg} \mathrm{Glp}$

c) Erythrozyten in Plasma + 10 bzw. $20 \mathrm{mg}$ GlpND

d) Erythrozyten in Plasma + ND (ND-Menge entsprach der NDMenge in GlpND)

e) Erythrozyten in Plasma + ND $100^{\circ}$

f) Erythrozyten in Plasma + GlpND $100^{\circ}$

Bestimmung der enzymatisch freizusetzenden Neuraminsäure der Erytbrozyten

In einem weiteren Versuchsansatz wurden $2 \mathrm{ml}$ gewaschene Erythrozyten in $10 \mathrm{~m} l$ physiol. Kochsalzlösung aufgeschwemmt. In den Ansätzen der physiol. $\mathrm{NaCl}$-Lösung waren die oben unter $a-f$ aufgeführten Substanzen enthalten. Nach $30 \mathrm{Min}$. wurden die Erythrozyten abzentrifugiert und im Uberstand die freie und gebundene Neuraminsäure ermittelt. Die gewaschenen Erythrozyten wurden mit dem Plasma desselben Patienten im Verhältnis 1:1 vereint und die Blutsenkungsreaktion angesetzt. Nach Senkungsablauf wurden die erneut gewaschenen Erythrozyten $0,5 \mathrm{~m} / \mathrm{ge}-$ packte Erythrozyten $+0,2 \mathrm{~m} l$ Neuraminidase ad $5,0 \mathrm{~m} l$ physiol. 
Kochsalzlösung, Substrat: Enzym-Verhältnis $1: 1)$ bei $37^{\circ} 12$ Stdn. inkubiert. In dem Uberstand wurde ebenfalls die freie und gebundenc Neuraminsäure nach Ausfällen von Haemolyseprodukten mit Carrez-Reagenz bestimmt (1).

\section{Bestimmung der Neuraminsäure}

a) Gesamtneuraminsäure: Resocinol-Salzsäure-Methcde nach SvENNERHOLM (2)

b) freie Neuraminsäure: Thiobarbitursäure-Methode nach WARREN (3)

\section{Ansetzen des Virus-Haemagglutinations-Testes}

Die Erythrozyten wurden in einer 10-proz. Aufschwemmung in physiol. Kochsalzlösung mit Grippe-Virus Typ Asia in 10-proz. Konzentration angesetzt ${ }^{1}$ ).

\section{Messung des Haemolyse-Grades}

Sie erfolgte im Sichtbaren bei 418 und im UV bei $280 \mathrm{~m} \mu$. Als Maß der Haemolyse wurde außerdem das aus den Erythrozyten freigesetzte Kalium flammenphotometrisch bestimmt.

\section{Ergebnisse}

\section{Abbängigkeit der Sedimentationsrate von der Glykoproteid- Konzentration}

Das in dieser Versuchsanordnung verwandte Glykoproteid Glp wurde aus dem Urin von Patienten mit malignen Tumoren isoliert (Präparation s. Tab. 1). Das Molekulargewicht beträgt 20-22000; die elektrophoretische Wanderungsgeschwindigkeit auf Papierund Membranfolien entspricht einem schnellen $\alpha_{1}$ Globulin; es ist in der Ultrazentrifuge homogen; der Peptidgehalt liegt $z$ wischen $55-58 \%$, der Kohlenhydrat-

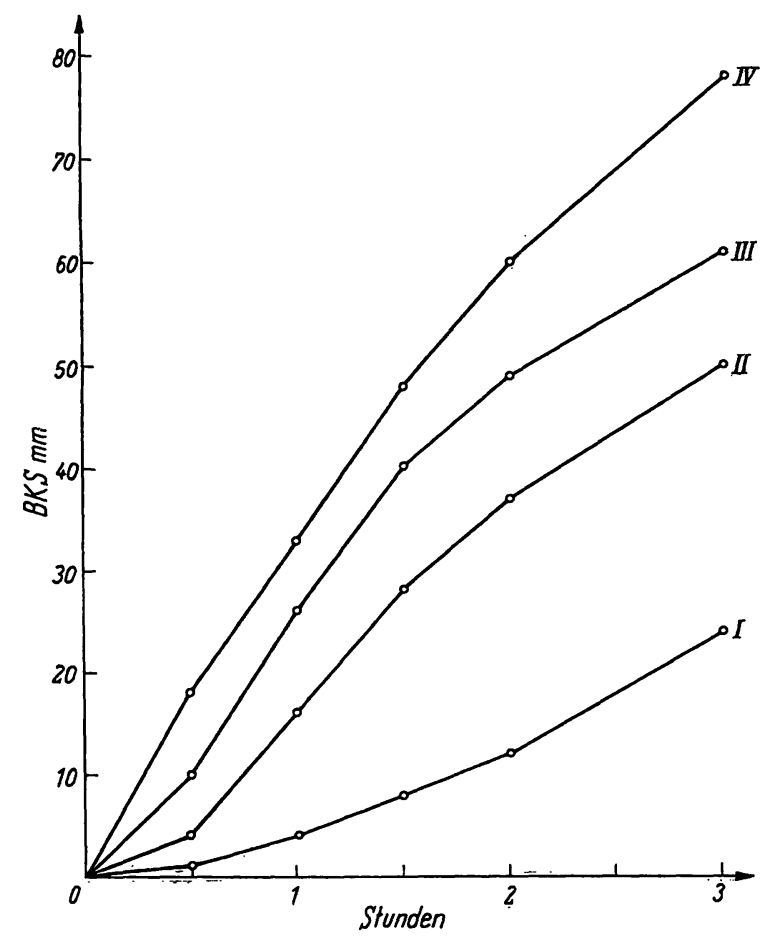

Abb. 1

Blutsenkungsreaktion nach Zusatz des Glykoproteids zu dem Plasma des Patientenblutes, $1 / 2$ stdg. Ablesung über 3 Stdn. $I=$ unbehandelte Vergleichsssenkung; $I I=$ nach Zusatz von 20 $\mathrm{mg}$; III = nach Zusatz von $30 \mathrm{mg}$; IV = nach Zusatz von $40 \mathrm{mg}$ Glykoproteid

1) Für die Untersuchungen danken wir Herrn Professor Dr. K. Binger, Direktor des Institutes für medizinische Virologie am Hygiene-Institut der Universität Heidelberg.

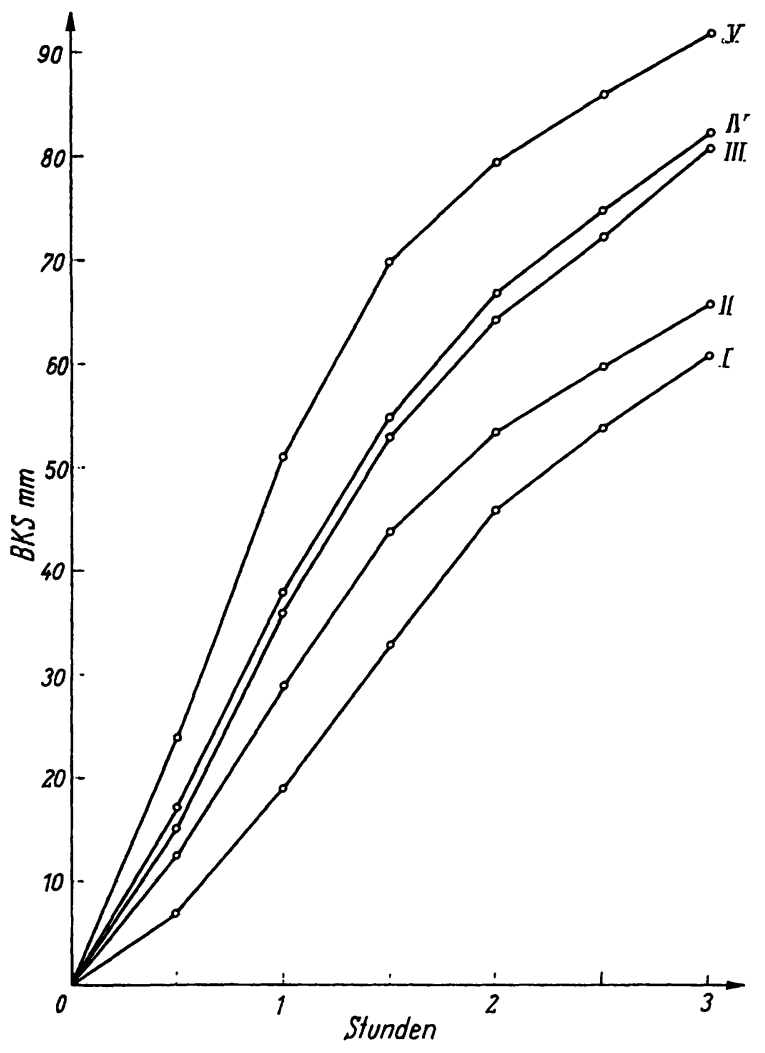

Abb. 2

Vergleichssenkung nach Zusatz von je $15 \mathrm{mg}$ Glykoproteid vor und nach Behandlung mit Neuraminidase und Inaktivierung der nicht dialysierbaren Neuraminidase durch Erhitzen auf $100^{\circ}$. $I=$ unbehandelte Vergleichssenkung; $\Pi=$ Zusatz von Neuraminidase (ND); III = Zusatz von unbehandeltem Glykoproteid (Glp); IV = Zusatz von Glykoproteid nach komplettem Neuraminsäure-Release und Erhitzen des Glykoproteid-NeuraminidaseGemisches auf $100^{\circ}\left(\right.$ GlpND $\left.100^{\circ}\right)$; V = Zusatz von Glykoproteid nach kompletter Neuraminsäure-Abspaltung und Dialyse (GlpND). Der Neuraminidase-Gehalt des Glykoproteids nach Inkubation entsprach der in II zugesetzten Neuraminidase

gehalt zwischen $40-43 \%$. Die Kohlenhydratkomponenten haben folgende Verteilung: Neuraminsäure 8-8,5\%, Hexosen $18-20 \%$, Fucose 3,4-4\%, Hexosamine $10-11 \%$, Xylose 2,0\%. Die Konzentration der mit Neuraminidase abspaltbaren Neuraminsäure beträgt $7,5 \%$ (4).

Dieses Glykoproteid ist hitzestabil und perchlorsäurelöslich. Sowohl bei dem Zusatz zur normalen als auch zur beschleunigten Blutsenkung löst es eine Erhöhung der Sedimentationsrate aus, die der Konzentration des zugegebenen Glykoproteids parallel ging (Abb. 1). Wurde das Glykoproteid (GlpND) in gleicher Konzentration zugegeben, nachdem vorher die enzymatisch freizusetzende Neuraminsäure mit Neuraminidase abgespalten worden war, dann war die Erhöhung der Sedimentationsrate noch ausgeprägter. Wurde die durch Dialyse nicht zu entfernende Neuraminidase durch Erhitzen auf $100^{\circ}$ inaktiviert, dann war die BKS etwas weniger beschleunigt als bei Verwendung der Substanz vor Erhitzen. Setzte man Neuraminidase allein in der gleichen Konzentration dem Plasma zu, dann konnte bei Zimmertemperatur ebenfalls eine Beschleunigung der BKS beobachtet werden (Abb. 2). Nach $4-5$ Stdn. bei 

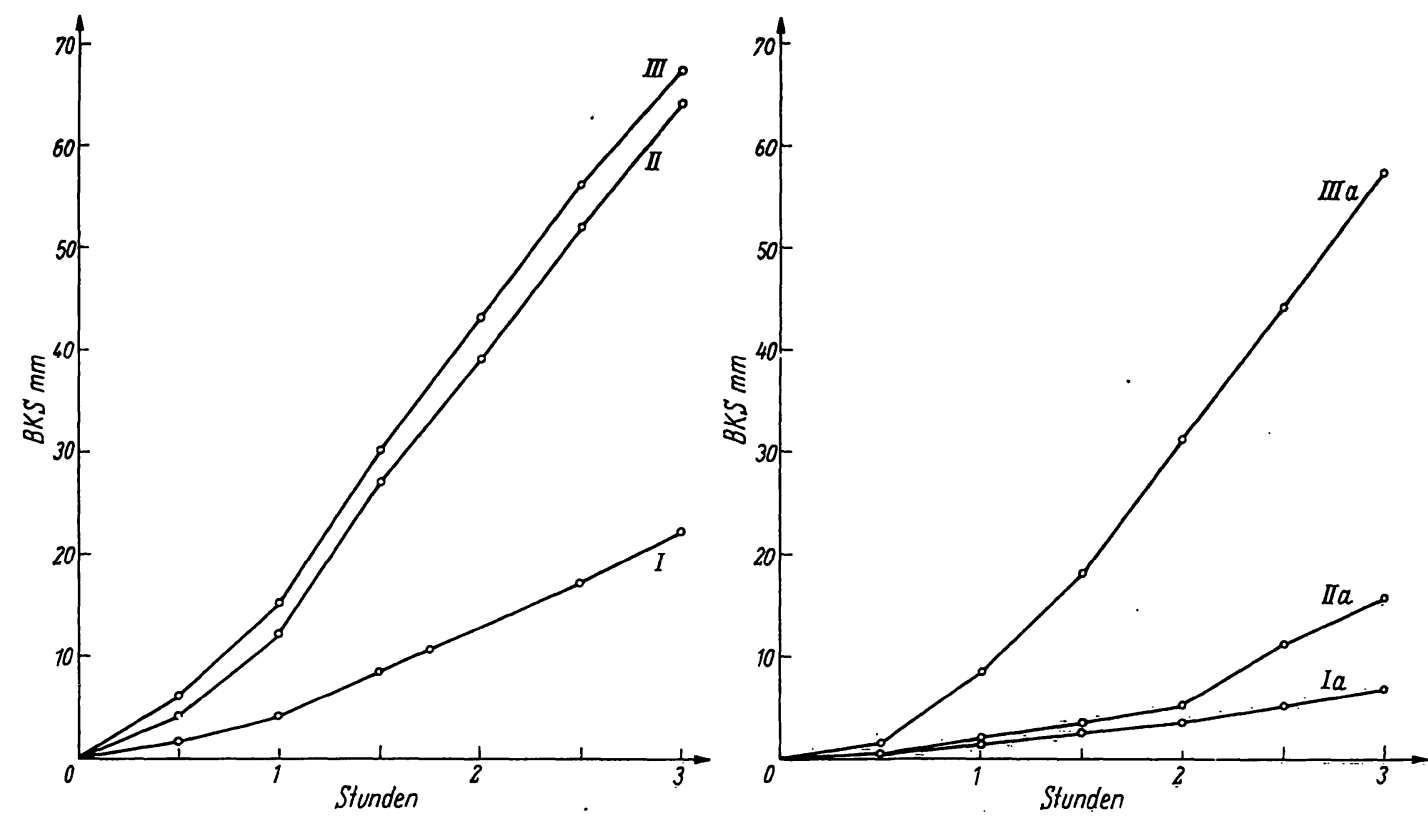

Abb. 3

Vergleichssenkung nach Zusatz von Gangliosiden vor und nach Inkubation mit Neuraminidase, die sich durch die enzymatisch abspaltbaren Neuraminsäure-Moleküle unterscheiden

$\mathrm{I}+\mathrm{Ia}=$ Senkung ohne Gangliosid-Zusatz. Zusatz von Gangliosid GGI I (es enthielt keine enzymatisch abspaltbare Neuraminsäure) II = vor und III = nacb Inkubation mit Neuraminidase. Zusatz von Gangliosid GGI II (es enthielt ein Molekül enzymatisch abspaltbarer Neuraminsäure) $\mathrm{II}_{\mathrm{a}}=$ nacb und $\mathrm{III} \mathrm{a}=$ vor Inkubation mit Neuraminidase

Zimmertemperatur oder nach kurzfristiger Inkubation bei $37^{\circ}$ war die Blutsenkung jedoch vollkommen gehemmt. Bei Verwendung des Glykoproteids (GlpND) blieb die BKS unverändert beschleunigt. Wurden anstelle des Glykoproteids (Glp) die Ganglioside (GGl I oder GGI II) verwandt, dann war ebenfalls eine Senkungsbeschleunigung zu beobachten. Nach NeuraminidaseInkubation war mit dem Gangliosid GGl I, das keine enzymatisch abspaltbare Neuraminsäure enthält, der gleiche Senkungsablauf zu beobachten. Bei dem Gangliosid GGI II, bei dem ein Molekül Neuraminsäure enzymatisch abspaltbar war, wurde die Blutsenkung nach Inkubation des Gangliosides mit Neuraminidase nicht beschleunigt (Abb. 3).

\section{Abbängigkeit der Sedimentationsrate von dem Erytbrozyten- Plasma-Volumenverbältnis}

Sowohl bei Gesunden als auch bei Patienten mit BKSBeschleunigung durch Dys- und Paraproteinämien mit unterschiedlicher proteingebundener Kohlenhydratkonzentration wurde durch die Variation der ErythrozytenPlasma-Relation die Sedimentationsrate stark beeinflußt. Durch Zunahme des Plasmaanteiles bei konstantem Serum-Eiweißgehalt war auch bei Normalpersonen ein Senkungsanstieg zu beobachten. Bei Plasma mit neuraminsäurereichen Glykoproteiden wurde dieser Effekt noch ausgeprägter. Wurde die Erythrozyten-Neuraminsäure vor dem Senkungsbeginn enzymatisch abgespalten; dann war die Beschleunigung durch Plasma-Volumenzunahme noch intensiver. Freie Neuraminsäure allein löste keine Senkungsbeschleunigung aus. Bei Polyglobulien konnte durch Vermehrung des Plasmaanteiles die normalerweise sonst vorliegende BKS-Hemmung aufgehoben werden. Erzielte man die Zunahme des
Plasma-Volumens dưch Zugabe von physiologischer Kochsalzlösung oder Natriumcitrat, so wurde schon bei einer geringen Plasmaverdünnung die BKS gehemmt. Konzentration des Plasma-Proteins bei gleichem Volumen führte zur Senkungsbeschleunigung (Tab. 2).

Enzymatische Abspaltung der Erytbrozyten-Neuraminsäure mit und obne Zusatz von Glykoproteiden oder Glykolipiden Von den mit physiologischer Kochsalzlösung gewaschenen Erythrozyten wurde $1 \mathrm{~m} l$ in physiologischer Kochsalzlösung aufgeschwemmt und mit $0,4 \mathrm{~m} l$ Neuraminidase $12 \mathrm{Stdn}$. bei $37^{\circ}$ und $\mathrm{pH}$ 5,7 inkubiert. In dem Überstand wurde die gesamte und die freie Neuraminsäure ermittelt. Der Haemolysegrad wurde im sichtbaren und im UV-Bereich gemessen. Das extrazelluläre Kalium, das dem Haemolysegrad parallel ging, wurde flammenphotometrisch ermittelt. Bei Erythrozyten von Normalpersonen lag die Gesamtneuraminsäure pro $\mathrm{m} l$ Erythrozyten zwischen 150 und $180 \mu \mathrm{g}$. 80-80 $\mu \mathrm{g}$ der Neuraminsäure konnten enzymatisch als freie Neuraminsäure abgespalten werden. Wurde zur Entfernung der Haemolyseprodukte der Úberstand mit Carrez-Reagenz ausgefällt, dann nahm die Gesamtneuraminsäure um $20 \%$ ab. Die Konzentration der freien Neuraminsäure ermittelt nach der Thiobarbitursäure-Methode blieb jedoch konstant. Unterschiede in dem Neuraminsäure-Release bei den einzelnen untersuchten Krankheitsbildern waren nicht nachzuweisen. Ebenso konnten keine verwertbaren Differenzen bei verschiedenen Blutgruppeneigenschaften des ABO- und MN-Systems festgestellt werden. Nach Blockierung der MN-Antigene durch MN-Antikörper war der Neuraminsäure-Release mit Neuraminidase um $30-40 \%$ herabgesetzt. Nach Zusatz von Glykoproteiden und Gangliosiden für 30-40 Min. zu -den 
Tab. 2

Blutsenkungsreaktion nach WESTERGREN im Vergleich zu unterschiedlichem Erythrozyten: Plasma-Verhältnis.

$a=$ Normalsenkung; $b=$ Erythrozyten: Plasma-Verhältnis $1: 1 ; c=$ Erythrozyten:Plasma-Verhältnis $1: 2 ; d=$ Einengung des Plasmas mit Dialysehülse auf den doppelten Proteingehalt, danach Ansetzen der Senkung im Erythrozyten:Plasma-Verhältnis von $1: 1$; $e=$ Plasmaverdünnung mit physiologischer Kochsalzlösung 1:1, danach Senkung Erythrozyten: Plasma-Verhältnis 1:1; Gesamteiweiß-Bestimmung; Zonenelektrophoretische Auftrennung auf Membranfolien; Berechnung der Gesamtglobuline; Bestimmung der Protein-gebundenen Kohlenhydrate: Gesamthexosen (Orcin- und Anthron-Reagenz), Neuraminsäure (Resorcinol-Salzsäure-Methode nach SVENNERIOLM), Methylpentosen (Cystein-HydrochloridMethode nach DISCHE und SHETTLES), Hexosamin (nach ELSON-MORGAN); Berechnung der gesamtproteingebundenen Kohlenhydrate zu den Gesamtglobulinen

\begin{tabular}{|c|c|c|c|c|c|c|c|c|c|c|c|c|c|c|c|c|c|}
\hline \multirow[t]{2}{*}{ Nr. } & \multirow[t]{2}{*}{ Name } & \multirow[t]{2}{*}{ Diagnose } & \multicolumn{5}{|c|}{$\begin{array}{c}\text { Blutkörperchensenkungsreaktion } \\
\text { in } \mathrm{mm} / \mathrm{Std} \text {. }\end{array}$} & \multirow{2}{*}{$\begin{array}{l}\text { Ges. } \\
\text { Ew }\end{array}$} & \multirow{2}{*}{\multicolumn{5}{|c|}{ Elektrophorese in rel. \% }} & \multirow{2}{*}{$\begin{array}{c}\text { Ges. } \\
\text { Glob. } \\
\%\end{array}$} & \multirow{2}{*}{ 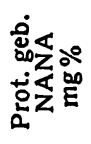 } & \multirow{2}{*}{ 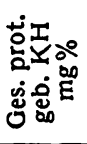 } & \multirow{2}{*}{ 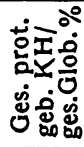 } \\
\hline & & & a & $\mathbf{b}$ & c & & e & & & & & & & & & & \\
\hline 1 & M.K. & gesund & $6 / 9$ & $1 / 2$ & $7 / 15$ & & $0 / 0$ & 6,3 & 62,0 & 3,2 & 7,8 & 12,7 & 14,3 & 38,0 & & & \\
\hline 2 & D.K. & gesund & $3 / 8$ & $1 / 2$ & $8 / 18$ & & $0 / 2$ & 6,3 & 61,9 & 3,3 & 7,7 & 12,8 & 14,3 & 38,1 & & & \\
\hline 3 & A. $\mathbf{T}$. & Leber-Ca & $41 / 71$ & $24 / 49$ & $67 / 112$ & & $2 / 4$ & 8,2 & 51,3 & 2,4 & 7,3 & 15,8 & 23,2 & 48,7 & 65 & 545 & 10,8 \\
\hline 4 & O.B. & Leber-Ca & $60 / 97$ & $38 / 70$ & $62 / 91$ & & $5 / 14$ & 8,2 & 51,2 & 2,6 & 7,3 & 15,6 & 23,3 & 48,8 & 65 & 545 & 10,8 \\
\hline 5 & F.B. & Bronchial-Ca & $63 / 82$ & $23 / 48$ & $90 / 124$ & & $0 / 2$ & 6,1 & 36,0 & 6,6 & 13,1 & 19,7 & 24,6 & 64,0 & 90 & 695 & 10,4 \\
\hline 6 & A.St. & Plasmocytom & $12 / 22$ & $5 / 15$ & $33 / 67$ & & $2 / 3$ & 7,2 & $\mathbf{5 7 , 0}$ & 4,2 & 15,2 & 12,5 & 11,1 & 43,0 & 70 & 580 & 13 \\
\hline 7 & D. K. & Plasmocytom & $118 / 121$ & $86 / 98$ & $127 / 131$ & $92 / 96$ & $4 / 6$ & 9,5 & 33,6 & 2,1 & 7,4 & 7,4 & 49,5 & 66,4 & 60 & 625 & 9,4 \\
\hline 8 & H. B. & Plasmocytom & $138 / 142$ & $108 / 117$ & $120 / 123$ & $84 / 90$ & $6 / 17$ & 9,4 & 38,3 & 4,3 & 8,5 & 8,5 & 40,4 & 61,7 & 58 & 516 & 8,3 \\
\hline 9 & O. H. & Plasmocytom & $8 / 28$ & $11 / 31$ & $54 / 93$ & $68 / 89$ & $2 / 3$ & 7,9 & 48,6 & 2,6 & 9,0 & 16,7 & 23,1 & 51,4 & & & \\
\hline 10 & O.D. & Plasmocytom & $43 / 71$ & $73 / 104$ & $78 / 107$ & $46 / 75$ & $3 / 6$ & 6,2 & 53,2 & 3,2 & 29,0 & 9,7 & 4,9 & 46,8 & & & \\
\hline 11 & A. $\mathbf{R}$. & Plasmocytom & $11 / 28$ & $10 / 16$ & $35 / 73$ & $44 / 79$ & - & 7,4 & 54,8 & 2,7 & 11,0 & 13,3 & 18,2 & 45,2 & 70 & 536 & 11,9 \\
\hline 12 & F. H. & Plasmocytom & $9 / 21$ & $3 / 5$ & $9 / 22$ & $7 / 15$ & $1 / 1$ & 5,6 & 60,6 & 5,4 & 10,7 & 12,5 & 10,8 & 39,4 & & & \\
\hline 13 & O.H. & Vasculitis & $28 / 59$ & $3 / 6$ & $33 / 66$ & - & $1 / 2$ & 4,3 & 34,8 & 4,7 & 11,6 & 16,3 & 32,6 & 65,2 & 44 & 460 & 7 \\
\hline 14 & D.H. & Polyarthritis & $11 / 24$ & $5 / 17$ & $26 / 59$ & $49 / 95$ & $2 / 3$ & 8,0 & 51,4 & 3,7 & 12,5 & 18,7 & 13,7 & 48,6 & 65 & 573 & 11,9 \\
\hline 15 & F.V. & Cholangitis & $26 / 54$ & $4 / 9$ & $42 / 69$ & $47 / 82$ & $1 / 2$ & 7,6 & 60,5 & 2,6 & 13,2 & 9,2 & 14,5 & 39,5 & & & \\
\hline 16 & K.D. & Pyelitis & $53 / 87$ & $36 / 62$ & $54 / 79$ & $62 / 82$ & $3 / 5$ & - & 56,4 & 1,9 & 8,6 & 10,5 & 22,6 & 43,6 & 61 & 666 & 15 \\
\hline 17 & K.St. & unklare BKS & $66 / 91$ & $25 / 38$ & $94 / 116$ & $70 / 77$ & $0 / 1$ & 7,7 & 38,2 & 4,0 & 10,5 & 21,0 & 26,3 & 61,8 & 56 & 638 & 10,3 \\
\hline 18 & F.W. & unklare BKS & $64 / 86$ & $51 / 67$ & $78 / 105$ & $65 / 75$ & $1 / 2$ & 7,2 & 41,6 & 5,6 & 9,7 & 11,1 & 32,0 & 58,4 & & & \\
\hline 19 & G.S. & Hodgkin & $65 / 102$ & $35 / 59$ & $82 / 113$ & - & $3 / 10$ & 6,2 & 33,8 & 8,1 & 21,0 & 16,1 & 21,0 & 66,2 & 126 & 892 & 13,3 \\
\hline 20 & A. $\mathrm{K}$. & Hodgkin & $15 / 26$ & $3 / 10$ & $18 / 58$ & $30 / 80$ & $1 / 2$ & 9,6 & 46,9 & 3,1 & 12,5 & 9,4 & 28,1 & 53,1 & & & \\
\hline 21 & F.N. & Perniciosa & $19 / 50$ & $2 / 4$ & $10 / 23$ & - & $0 / 2$ & 5,67 & 60,7 & 3,6 & 7,1 & 14,3 & 14,3 & 39,3 & 40 & 358 & 9,5 \\
\hline 22 & K. H. & FE-Anämie & $17 / 35$ & $2 / 3$ & $18 / 42$ & $4 / 13$ & $0 / 1$ & 8,6 & 51,9 & 3,5 & 9,4 & 9,4 & 25,8 & 48,1 & 71 & 614 & 12 \\
\hline 23 & A.B. & Polyglobulie & $1 / 2$ & $2 / 3$ & $9 / 21$ & - & $1 / 1$ & - & - & - & - & - & - & - & & & \\
\hline 24 & J.H. & haem. Anämie & $8 / 20$ & $3 / 5$ & $13 / 25$ & $26 / 45$ & $0 / 0$ & 8,1 & 51,9 & 4,9 & 9,9 & 16,0 & 17,3 & 51,9 & 70 & 522 & \\
\hline 25 & O. F. & Emphysem & $2 / 4$ & $13 / 24$ & $36 / 71$ & $55 / 92$ & $0 / 1$ & 7,6 & 58,4 & 3,9 & 9,1 & 11,7 & 16,9 & 41,6 & 68 & 567 & 14 \\
\hline 26 & F.F. & Myotonie & $7 / 31^{\circ}$ & $3 / 8$ & $20 / 41$ & $32 / 54$ & $0 / 2$ & 6,5 & 64,0 & 3,1 & 11,0 & 7,8 & 14,1 & 36,0 & 60 & 505 & 14,5 \\
\hline
\end{tabular}

Erythrozyten und anschließendem Waschen wurde durch das Glykoproteid (Glp) und die Ganglioside (GGl I und GGI II) der Neuraminsäure-Release der Erythrozyten nicht beeinflußt. Hingegen konnte nach Zugabe von dem Glykoproteid (GlpND) auch bei nachfolgender optimaler Inkubation mit Neuraminidase bei $37^{\circ}$ (12 Stdn., pH 5,7, Substrat: Enzym-Verhältnis = 10:1) keine Neuraminsäure mehr von den Erythrozyten abgespalten werden. Waren die Erythrozyten unter gleichen Ver- suchsbedingungen nur mit Neuraminidase allein behandelt worden, dann konnte mit nachfolgender optimaler Neuraminidase-Inkubation noch $20-30 \%$ Erythrozyten-Neuraminsäure freigesetzt werden. Wurde hitzeinaktiviertes Glykoproteid (GlpND $100^{\circ}$ ) oder Neuraminidase (ND $100^{\circ}$ ) in dem Versuchsansatz verwandt, dann war durch optimale Neuraminidase-Inkubation die gleiche Neuraminsäure-Quote enzymatisch freizusetzen wie bei unbehandelten Erythrozyten (Tab.3).

Tab. 3

BKS-Kontrolle bei verschiedenen Neuraminidase-Inkubations-Zeiten und Neuraminidase-Mengen.

$I=$ Substrat: Enzym-Verhältnis 10:1; II = Substrat: Enzym-Verhältnis 10:2; III = Vergleichssenkung ohne Neuraminidase-Zusatz

\begin{tabular}{|c|c|c|c|c|c|c|c|c|}
\hline \multicolumn{3}{|c|}{ I } & \multicolumn{3}{|c|}{ II } & \multicolumn{3}{|c|}{ III } \\
\hline$\underset{\mathrm{mm} / \mathrm{Std}}{\mathrm{BKS}}$ & $\begin{array}{l}\text { NANA }_{S \mathrm{~V}} \\
\mu \mathrm{g} / \mathrm{m} l\end{array}$ & $\begin{array}{c}\text { NANAw } \\
\text { Erythrozyten }\end{array}$ & $\begin{array}{c}\text { BKSS } \\
\mathrm{mm} / \mathrm{Std} .\end{array}$ & $\begin{array}{l}\text { NANA }_{S V} \\
\mu \mathrm{g} / \mathrm{m} l\end{array}$ & $\begin{array}{l}\text { NANAw } \\
\text { Erythrozyten }\end{array}$ & $\begin{array}{c}\text { BKS } \\
\mathrm{mm} / \mathrm{Std} .\end{array}$ & $\begin{array}{l}\text { NANA }_{S \nabla} \\
\mu \mathrm{g} / \mathrm{m} l\end{array}$ & $\begin{array}{c}\text { NANAw } \\
\text { Erythrozyten }\end{array}$ \\
\hline
\end{tabular}

nach 30 Minuten Inkubation bei Zimmertemperatur

\begin{tabular}{|c|c|c|c|c|c|}
\hline $\begin{array}{l}1,5 \\
4 \\
6,5\end{array}$ & 54,0 & 39,6 & $\begin{array}{c}7,5 \\
12 \\
15\end{array}$ & 63,0 & 37,8 \\
\hline
\end{tabular}

nach 180 Minuten Inkubation bei Zimmertemperatur

4

8

1,5
3

99,0

58,4

6,5

117,0

78,3

2,5

5,0

0

nach 12 Stunden Inkubation bei $37^{\circ}$

\begin{tabular}{llllll}
4,5 & & & 0,5 & 0 \\
7 & 171,0 & 127,8 & 1 & 0 & \\
9 & & & 2 & & \\
\hline
\end{tabular}


Tab. 4

Behandlung der Etythrozyten mit Neuraminidase (ND), hitzeinaktivierter Neuraminidase (ND $100^{\circ}$ ), Neuraminidase-behandeltem Glykoproteid (GlpND), Neuraminidase-behandeltem Glykoproteid nach Hitzeinaktivierung (GlpND $100^{\circ}$ )

Die Einzelsubstanzen wurden in physiol. Kochsalzlösung gelöst $(15 \mathrm{mg} / 10 \mathrm{ml})$, danach Herstellung einer Erythrozyten-Suspension von $2 \mathrm{~m} l$ Erythrozyten in $10 \mathrm{~m} l$ physiol. Kochsalzlösung, nach 30 Min. Abzentrifugation der Erythrozyten, Ansetzen der Senkung mit $1 \mathrm{ml}$ Erythrozyten $+1 \mathrm{ml}$ Plasma desselben Patienten, Senkungsablesung nach 1 und 2 Stdn. Neuraminsäure-Bestimmung in dem Ưberstand nach Zentrifugation mit der Thiobarbitursäure-Bestimmung (WARREN). Eneute Inkubation derErythrozyten mit ND, 12 Stdn., $37^{\circ}$, Substrat: Enzym-Verhältnis 10:1, pH 5,7. Ansetzen des Virus-Haemagglutinations-Testes nach 30 Min. Inkubation, MN-Blutgruppenbestimmung nach 12 Stdn. ND-Inkubation

\begin{tabular}{|c|c|c|c|c|c|}
\hline & ND & ND $100^{\circ}$ & GIpND & GlpND $100^{\circ}$ & Normalsenkung \\
\hline BKS & $4 / 7$ & $7 / 16$ & $14 / 28$ & $9 / 20$ & $5 / 14$ \\
\hline $\begin{array}{l}\text { NS-Release nach } 30 \mathrm{Min} \text {. Inkubation bei } 20^{\circ} \\
\text { NS-Release nach nochmaliger Inkubation }\end{array}$ & 105 & 0 & 100 & $\emptyset$ & $\emptyset$ \\
\hline $\begin{array}{l}12 \mathrm{Stdn} ., 37^{\circ}, \mathrm{pH} 5,7 \\
\text { Virus-Haemagglutinat. Test nach } 30 \mathrm{Min} .\end{array}$ & 35 & 140 & $\emptyset$ & 100 & 140 \\
\hline $\begin{array}{l}\text { Inkubation bei } 20^{\circ} \\
\text { MN-Blutgruppeneigenschaften nach opti- }\end{array}$ & $1: 512$ & $1: 2048$ & $1: 2$ & $1: 2048$ & $1: 2048$ \\
\hline maler Inkubation 12 Stdn., $37^{\circ} \mathrm{pH} 5,7$ & $\mathbf{N}$ & $\mathrm{N}$ & MN & $\mathbf{N}$ & $\mathbf{N}$ \\
\hline
\end{tabular}

Beeinflussung der Virus-Haemagglutination und der MN-Blutgruppeneigenschaften

Das Glykoproteid wurde 40 Min. der Erythrozyten-Aufschwemmung in physiologischer Kochsalzlösung zugegeben. Danach wurden die Erythrozyten intensiv gewaschen und in einer 10-proz. Aufschwemmung in physiologischer Kochsalzlösung zum Virus-Haemagglutinations-Test verwandt. Parallel zu der Sedimentationsrate nach Zugabe des Glykoproteids (GlpND) und dem rerminderten enzymatischen Neuraminsäure-Release konnte mit dieser Substanz auch die VirusHaemagglutination komplett gehemmt werden. Die Hemmung ging der GlpND-Konzentration parallel. Bei Verwendung von Neuraminidase unter gleichen Versuchsbedingungen konnte keine HaemagglutinationsHemmung erzielt werden. Bei hitzeinaktiviertem Glykoproteid (GlpND $100^{\circ}$ ) und dem nicht enzymatisch vorbehandelten Glykoproteid (Glp) trat kein Hemm-Effekt ein. Die Beeinflussung der MN-Blutgruppeneigenschaften durch intensive Inkubation mit Neuraminidase - über die an anderer Stelle ausführlich berichtet wird konnte durch das Glykoproteid (GlpND) ebenfalls ausgeschaltet werden (Tab. 4).

\section{Diskussion}

In den Untersuchungsergebnissen von RUHENSTROTHBAUER und Mitarbeitern $(5,6,7)$ konnte gezeigt werden, $\mathrm{da} B$ einzelne Plasmafaktoren, bezeichnet als Agglomerine, den Senkungsmechanismus wesentlich beeinflussen. Gegenüber diesen Proteinen sollen die ErythrozytenFaktoren und die elektrostatische Membranladung weniger bedeutungsvoll sein. Substanzen, die unter dem Begriff der Sedimentine von diesen Autoren zusammengefaßt wurden, sind ebenfalls für den Senkungsablauf wichtig. Durch das Vorkommen natürlicher senkungshemmender Substanzen wie einzelner Fettsäuren und Glykolipide (8) sowie die Beeinflussung der Sedimentationsrate durch Agglomerationsblocker oder Teilblocker wie Butazolidin, Steroide und andere mehr wird die Deutung des globalen Vorgangs dieser Routine- methode recht schwierig. STARY und Mitarbeiter (9) zeigten' anhand entzündlicher Erkrankungen mit $\alpha_{1}$ und $\alpha_{2}$-Vermehrung den senkungsbeschleunigenden Effekt der kohlenhydratreichen Glykoproteide. STICKL und BöckER (10) konnten die Bedeutung der Erythrozyten-Plasma-Neuraminsäure-Relation in ihren Untersuchungen als wesentlich für den Blutsenkungs-Ablauf demonstrieren. In der hier beschriebenen Versuchsanordnung konnte gezeigt werden, $\mathrm{da} B$ sowohl die normale als auch die pathologisch beschleunigte BKS durch Zusatz eines Glykoproteids mit den chemischen und physikalischen Eigenschaften, die dem sauren $\alpha_{1}$-Glykoproteid sehr ähnlich sind, konzentrationsabhängig beschleunigt wurde (Abb. 1).

Wurde dem Plasma Gangliosid zugesetzt, so löste dieses neuraminsäurehaltige Glykolipid ebenfalls eine $\mathrm{Zu}$ nahme der Sedimentationsrate aus. Bei gleichzeitiger schonender enzymatischer Abspaltung der Erythrozyten-Neuraminsäure wurde der Glykoproteid- und Glykolipid-Effekt auf die BKS noch deutlicher. Hieraus ist zu ersehen, daß die Korrelation der ErythrozytenMembranladung.$z u$ der negativen Plasma-Ladung, die durch neuraminsäurehaltige Glykolipide und Glykoproteide zunimmt, entscheidend für die Senkungsgeschwindigkeit sein kann. Durch die enzymatische Abspaltung der Erythrozyten-Neuraminsäure allein ist dieser Sachverhalt schon zu demonstrieren. Hierbei ist jedoch entscheidend, daß kein kompletter Neuraminsäure-Release stattgefunden hat wie es bei optimaler Inkubation mit Neuraminidase bei $37^{\circ}$ der Fall ist, da danach durch die intensive Erythrozyten-Membranveränderung eine totale Senkungshemmung eintritt. Auch die Erhöhung der Sedimentationsrate durch $\mathrm{Zu}-$ nahme des Plasma-Volumens bei konstanter PlasmaProtein-Konzentration dürfte durch die Zunahme der senkungsbeschleunigenden Plasma-Faktoren pro Erythrozyt bedingt sein. Hingegen führt eine Hydrämie - ausgelöst durch eiweißfreie Flüssigkeitszunahme des Plasmas oder eine Erythrozyten-Volumen-Vermehrung zur Reduktion der Sedimentationsrate. Schwieriger hin- 
gegen ist zu erklären, weshalb das Glykoproteid GlpND, nachdem die enzymatisch abspaltbare Neuraminsäure durch Neuraminidase entfernt worden war, die Blutsenkung stärker beschleunigt als das neuraminsäurehaltige Glykoproteid. Aufgrund der Untersuchungsergebnisse nimmt die Erythrozyten-Agglomeration in Gegenwart von neuraminsäurehaltigen Glykoproteiden und Glykolipiden im Plasma durch relative Abnahme der Erythrozyten-Membranladung zu. Ein Teil der zusätzlich verminderten Membranladung dürfte auf die Wirkung der Neuraminidase, die in dem Glykoproteid-Komplex (GlpND) vorhanden ist, zurückzuführen sein. $\mathrm{Da}$ diese Substanz jedoch die Sedimentationsrate stärker beschleunigt als Neuraminidase in gleicher Plasma-Konzentration und das hitzeinaktivierte Glykoproteid (GlpND $100^{\circ}$ ) ist es naheliegend, daß das Glykoproteid (GlpND) nachdem die Erythrozyten-Neuraminsäure enzymatisch partiell abgespalten wurde, mit der Erythrozyten-Membran eine agglomerationssteigernde Verbindung eingeht. Hierfür spricht auch die Beobachtung, daß das Glykoproteid (GlpND) durch intensives Waschen nicht von den Erythrozyten getrennt werden kann, die Virus-Haemagglutination komplett gehemmt wird und durch zusätzliche optimale Inkubation mit Neuraminidase keine weitere Neuraminsäure mehr abgespalten werden kann. Diese Substanz zeigt aufgrund sterisch bedingter Veränderungen nach der Behandlung mit Neuraminidase ein ähnliches Verhalten an der Erythrozyten-Membran wie wir es bei Antikörper-Reaktionen (11) finden, die ebenfalls zu einer starken Erhöhung der Sedimentationsrate und einer Verminderung des Neuraminsäure-Releases führen können. $\mathrm{Da}$ das Gangliosid (GGI II ND) keinen Anstieg der Blutsenkung auslöste, dürfte dem Glykoproteid-Charakter, unabhängig von dem Neuraminsäure-Gehalt, in der Erythrozyten-Agglomerisation eine spezielle Bedeutung zukommen.

Aus diesen Untersuchungen ergeben sich außerdem einige praktisch wichtige Schlußfolgerungen. Die neuraminsäurehaltigen Glykoproteide-spielen sicherlich eine wesentliche Rolle in dem Ablauf des Senkungsmechanismus, da durch ihren sauren Charakter die negative Plasma-Ladung erhöht wird, die zu einer Steigerung der Erythrozyten-Agglomerisation führt. Dies dürfte die Ursache der Senkungsbeschleunigungen bei Patienten mit $\alpha_{1}$ - und $\alpha_{2}$-Globulin-Vermehrung im wesentlichen bedingen. Ein schonender Erythrozyten-Neuraminsäure-Release der Erythrozyten - wie er bei längerem Stehen des Blutes durch bakteriell bedingte enzymatische Neuraminsäure-Freisetzung eintreten kann - kann ebenfalls zu einer Ethöhung der Sedimentationsrate durch Verminderung der negativen ErythrozytenLadung führen. Die Plasma-Volumenzunahme führt zu einer Konzentrationssteigerung der Agglomerine pro Erythrozyt, die ebenfalls eine Zunahme der BKS bedingt. Besonders in den Fällen, in denen neuraminsäurereiche Glykoproteid-Fraktionen im Serum vorliegen, tritt dann eine starke Erhöhung der Sedimentationsrate ein.

Erythrozyten-Volumenzunahme bei Polyzythämien und Bluteindickung reduziert durch Abnahme des PlasmaVolumens die Sedimentationsrate. Ebenfalls können ganz geringe Änderungen der Natriumcitrat-Konzentration bei der Blutsenkungs-Entnahme die BKS hemmen. Hydrämien, bei denen eiweißfreie Flüssigkeitsvermehrungen des Plasmas vorliegen, wie es $z$. B. bei Ödem-Ausschwemmungen vorübergehend der Fall sein kann, vermindern ebenfalls die ErythrozytenSenkungsgeschwindigkeit. Neben den oben erwähnten Senkungsblockern auf medikamentöser Basis können diese Faktoren, die mit der krankheitsbedingten Senkungsbeschleunigung in keiner ursächlichen Verbindung stehen, die Bewertung dieser Routine-Methode erschweren. $\mathrm{Da}$ oft in der Verlaufsbeurteilung eines Krankheitsbildes - bei differentialdiagnostischen Erwägungen und Bewertung der Therapieerfolge - die Blutsenkung herangezogen wird, erscheint es wichtig, diese Fehlerquellen zu kennen, damit in Zweifelsfällen eine zusätzliche Serum-Eiweißdifferenzierung mit anderen Methoden angewandt wird.

Obwohl die BKS seit vielen Jahren zu den meistgeübtesten Routine-Methoden gehört, die trotz aller modernen Eiweiß-Differenzierungen praktisch unvermindert in Klinik und Praxis angewandt wird, ist es wichtig zu wissen, daß ihre Aussagekraft nicht nur beschränkt wird aufgrund ihrer einfachen Technik, sondern auch durch die vielen Reaktionskonstellationen, die sich bei dieser nach wie vor genialen Methode in einem Röhrchen innerhalb von 2 Stunden abspielen.

Für die Untersuchungen der MN-Faktoren danken wir Frau Dr. I. KLose, Institut für gerichtliche Medizin der Universität Heidelberg.

\section{Literatur}

1. Tischr, I. und D. Peters, Żbl. Bakteriol. Abt. I. Orig. 195, 408 (1965). - 2. Svennirholm, L., Biochem. biophysica Acta (Amsterdam) 24, 604 (1957). - 3. WARREN, L., J. biol. Chemistry 234, 1971 (1959). - 4. Wercker, H. und K. Huhnstock, Klin. Wschr. 43, 1246 (1965). - 5. RuHenstroth-BAuer, G., F. H. KAYSER und G. NASS, Behring-Werke Mitteilungen Nr. 42, 41 (1962). - 6. Ruhenstroth-Bauer, G., G. Brittinger, F. H.
Kayser, G. Nass und M. TAutfest, Klin. Wschr. 40, 1200 (1962). -7. Ruhenstroth-Bauer, G., Klin. Wschr. 43, 460 (1965). - 8. ERLY, A. M., F. Gramlich und H. E. MülLer, Klin. Wschr. 43, 943 (1965). - 9. StaRY, Z., H. Bouder und F. BatrYoK, Schweiz. med. Wschr. 81, 1273 (1951). - 10. SrickL, H. und H. BöCKER, Klin. Wschr. 37, 635 (1959). - 11. JeAnNet, M. und A. Hässig, Blut 10, 297 (1964). 